\title{
Marchas y contramarchas de la educación técnica durante el tercer gobierno peronista (1973-1976)
}

Marches and countermarches of technical education during the third Peronist government (1973-1976)

Marchas e contramarchas da educação técnica durante o terceiro governo peronista (1973-1976)

\author{
Gabriela Andrea D'Ascanio \\ Universidad Nacional de Rosario (Argentina) \\ Consejo Nacional de Investigaciones Científicas y Técnicas \\ https://orcid.org/0000-0001-9809-3820 \\ gabidascanio@gmail.com
}

\section{Resumen}

Este artículo reconstruye los avatares de la educación técnica nacional entre 1973 y 1976. Se analizan los discursos y proyectos de reformas del sistema educativo y las acciones del Consejo Nacional de Educación Técnica (CONET). Se desarrolla la hipótesis de que, pese a que la presidencia del CONET estuvo a cargo del mismo funcionario durante todo el período, pueden advertirse dos momentos diferenciados. Durante la gestión de Taiana como ministro de Educación hubo especial interés en vincular la educación y el trabajo, en desarrollar innovaciones institucionales e introducir cambios en la estructura curricular del nivel medio técnico. Luego de la muerte del Presidente Perón, y de la sustitución de este funcionario, las preocupaciones por el control y la disciplina concentraron la atención, limitando el alcance de los proyectos transformadores relacionados con la oferta educativa del CONET. La base empírica del texto está compuesta por documentación oficial y fuentes periodísticas.

Palabras claves: Educación Técnica Nacional. CONET. Peronismo. 


\begin{abstract}
This article reconstructs the vicissitudes of the national technical education between 1973 and 1976. The speeches and reform projects of the educational system and the actions of the National Council for Technical Education (CONET) are analyzed. The developed hypothesis is that, although the presidency of CONET was in charge of the same official throughout the period, two different moments can be observed. During Taiana's tenure as Minister of Education, there was a special interest in linking education and work, in developing institutional innovations and introducing changes in the curricular structure of the technical middle level. After the death of President Perón, and the replacement of this official, the government focused the attention on control and discipline concerns, limiting the scope of transformative projects related to CONET's educational offerings. The empirical base of the text is composed of official documentation and journalistic sources.
\end{abstract}

Keywords: National Technical Education. CONET. Peronism.

\title{
Resumo
}

Este artigo reconstrói os altos e baixos da educação técnica nacional entre 1973 e 1976. São analisados os discursos e projetos de reforma do sistema educacional e as ações do Conselho Nacional de Educação Técnica (CONET). Desenvolve-se a hipótese de que, apesar da presidência do CONET ter estado a cargo do mesmo governante ao longo do período, dois momentos distintos podem ser observados. Durante o mandato de Taiana como Ministra da Educação, houve um interesse especial na articulação da educação e do trabalho, no desenvolvimento de inovações institucionais e na introdução de mudanças na estrutura curricular do nível técnico médio. Após a morte do Presidente Perón e a substituição deste governante, as preocupações de controle e disciplina centraram as atenções, limitando o âmbito dos projetos transformadores relacionados com a oferta educativa do CONET. A base empírica do texto é composta por documentação oficial e fontes jornalísticas.

Palavras-chave: Educação Técnica Nacional. CONET. Peronismo. 


\section{Introducción}

Los gobiernos justicialistas que se sucedieron desde el 25 de mayo de 1973 al 23 de marzo de 1976 suscitaron acciones y políticas antagónicas a lo largo del período. Tras siete años de dictadura militar, luego de un decenio de gobiernos con representatividad limitada por la proscripción del peronismo, éste volvió a ganar las elecciones nacionales canalizando diversas expectativas sociales. El pacto social que precedió a los comicios de 1973 y su ratificación en el Gran Acuerdo Nacional que tuvo lugar una vez llegado al poder el Frente Justicialista de Liberación (FREJULI), encabezado por Héctor J. Cámpora, que respondía a los lineamientos que Juan Domingo Perón establecía desde el exilio, permitió a un sector mayoritario de los argentinos pensar en el retorno a la idea de "comunidad organizada" propuesta por la doctrina peronista (LEYBA, 2003). Tras la renuncia de Cámpora y luego del breve gobierno provisional de Raúl Lastiri -presidente de la Cámara de Diputados de la Nación, en nuevas elecciones, el líder de este movimiento político llegó al poder ese mismo año, manteniendo una política de integración en un escenario de múltiples presiones sectoriales y demandas redistributivas resultantes de las alianzas promovidas por el gobierno (ROUGIER Y FISZBEIN, 2006). Dentro del movimiento peronista, la presencia de tendencias revolucionarias, expresadas en el grupo armado Montoneros, tuvieron como respuesta el paso a la clandestinidad de este colectivo, en mayo de 1974. La imprevista muerte del presidente Perón y la asunción de María Isabel Martínez de Perón -su esposa y vicepresidenta de la Nación- implicó un viraje de las políticas estatales hacia posiciones de derecha (FRANCO, 2011; 2012; LENCI, 1998; 1999; 2014), que se oponía en casi todos los aspectos al plan de gobierno propuesto por Cámpora en mayo de 1973. La heterogeneidad ideológica, la repentina sucesión de funcionarios y la complejidad del marco socio-político y económico condensaron sentidos y propuestas divergentes, que rápidamente disolvieron la homogeneidad del movimiento constituida durante las décadas de 1940 y 1950 (REIN, 1998). La lucha armada y la violencia política se constituyeron en canales a través de los cuales se expresaron los antagonismos. Los años del tercer gobierno peronista, como indican Rougier y Fiszbein (2006, p. 9), fueron algunos de los más "vertiginosos, controvertidos y complejos" de la historia contemporánea Argentina.

Durante los primeros gobiernos peronistas gran parte de las políticas educacionales se orientaron a fortalecer el vínculo entre educación y trabajo industrial en el marco de un proyecto de política económica que buscaba afianzar la industrialización nacional para hacer frente a la insuficiencia de stock provocada por la Segunda Guerra Mundial. El subsistema de educación técnica se consolidó a partir de la creación -en 1944- de la Comisión Nacional de Orientación y Aprendizaje Profesional (CNAOP), órgano autárquico que fue sustituido por el Consejo Nacional de Educación Técnica en 1959. Este debía dirigir y administrar los establecimientos de educación técnica y formación profesional que habían dependido de la CNAOP, así como del Ministerio de Cultura y Educación de la Nación. Su misión fue la de "educar integralmente a la juventud y lograr la capacitación técnico profesional de sus educandos." Le fueron transferidas las partidas que antiguamente se le asignaban a la CNAOP incluido el impuesto para el aprendizaje. ${ }^{2}$ A mediados de la década de 1960 se llevó adelante una unificación institucional de todas las escuelas medias que dependían del CONET, bajo la denominación de escuelas nacionales de educación técnica (ENETs), suprimiéndose las escuelas-fábricas y las escuelas industriales de la nación (PINEAU,

\footnotetext{
${ }^{1}$ Boletín Oficial, Ley 15.240/59, año LXVII, no. 19.055 (1959).

${ }^{2}$ El impuesto para el aprendizaje era una obligación tributaria de las empresas industriales que representaba un ingreso al CONET del $0.1 \%$ de lo que cada firma pagaba en salarios. Los industriales podían optar por hacer el aporte en forma directa al organismo o bien subvencionar escuelas técnicas o cursos profesionales privados aprobados por el CONET.
} 
1997a). Las primeras habían se habían constituido junto a la CNAOP y constaban de dos ciclos correspondientes a la enseñanza media con régimen mixto de enseñanza y producción. Las escuelas industriales de la nación, por su parte, eran establecimientos dependientes del Ministerio de Educación cuyas propuestas curriculares para el nivel medio, según los empresarios nucleados en la Unión Industrial Argentina, tenían una impronta teórica muy marcada que obstaculizaba la formación para el desempeño al interior de las industrias (PRONKO, 2009). Desde entonces, el nivel medio técnico nacional mantuvo una estructura curricular organizada en dos ciclos de tres años cada uno, que exigían asistencia matutina y vespertina. El primero era común a todas las especialidades y propendía tanto a la formación integral del educando como al contacto directo con las actividades productivas y laborales. Tras su finalización, el alumno podía optar por cursar el "cuarto año de término" o continuar el ciclo superior. En el primer caso, obtenía el título de Auxiliar Técnico en una especialidad dado que el plan de estudios enfatizaba en la cultura profesional con aplicaciones prácticas, para formar un operario calificado. El ciclo superior consistía en un complemento teóricocientífico que permitía adquirir una especialización para conducir el proceso laboral.

Los gobernantes desarrollistas que se sucedieron a lo largo de la década de 1960 destacaron con frecuencia las potencialidades de la educación, y de la educación técnica en particular, como dinamizadoras de la modernización tecnológica, económica y social. De tal modo, la educación técnica aparecía en un lugar privilegiado en el proceso de cambio que se proclamaba, cuya meta era lograr una industrialización pesada, un deseo incumplido en las dos décadas anteriores. No obstante, el impulso de este subsistema de enseñanza no fue uniforme, de modo que no llegaron a concretarse las ideas que lo promovían. Este tema ha recibido una relativa atención de los autores en el período siguiente.

El objetivo de este artículo es reconstruir el desarrollo de la educación técnica nacional durante el tercer gobierno peronista mediante el análisis de los discursos y los proyectos de reformas del sistema educativo formulados por los ministros y funcionarios estatales, así como de las políticas educacionales del CONET con relación a sus ENETs. Se parte de la hipótesis de que, pese a que la presidencia del CONET estuvo a cargo del mismo funcionario durante todo el período, pueden advertirse dos momentos diferenciados. Durante la gestión de Jorge Alberto Taiana como ministro de Educación hubo especial interés en vincular la educación y el trabajo, en desarrollar innovaciones institucionales e introducir cambios en la estructura curricular del nivel medio técnico. Luego de la muerte del presidente Perón, las preocupaciones por el control y la disciplina concentraron la atención, limitando el alcance de los proyectos transformadores de la oferta educativa del CONET. No obstante, se introdujeron políticas diferentes a las diseñadas durante el ministerio de Taiana que, de alguna forma, intentaron promover el vínculo educación-trabajo.

En este artículo se parte de la noción de que el Estado no es un actor unívoco sino un espacio polifónico en el que se relacionan, expresan e intervienen funcionarios y agencias diversas, provocando continuos reacomodamientos en su interior (BOHOSLAVSKY Y SOPRANO, 2010). En este sentido, las instituciones gubernamentales y burocráticas son entendidas como actores dinámicos, que intervienen en los procesos políticos generando tensiones y confrontaciones entre sí y con las agendas públicas, las políticas y la sociedad civil (OSZLAK, 2006). Aportando a esta idea, se describen en este texto algunos rasgos principales de las trayectorias personales e ideas de los funcionarios, en articulación con la marcha de las políticas estatales, tal como lo proponen Ben Plotkin y Zimmermann (2012).

En el campo disciplinar de la Historia de la Educación Argentina existe una producción considerable sobre los dos primeros gobiernos peronistas. Respecto del subsistema de educación técnica que se creó dependiente de la CNAOP se destacan los trabajos de Dussel y Pineau (2002) y de Koc Muñoz (2014). El nivel medio de la modalidad técnica ha sido estudiado por Pineau (1991) quien recuperó la investigación de Wiñar (1981) 
y le aportó otros indicadores empíricos con los cuales reveló la complejidad política del desarrollo institucional de la enseñanza técnica, focalizando en la aparición y funcionamiento de las escuelas-fábricas. Respecto al nivel universitario dependiente de la CNAOP son sugerentes los artículos de Pineau (1997a) y Mollis (1991). El primero abordó las interpretaciones que distintos académicos dieron a la Universidad Obrera Nacional y el segundo, centrado en las particularidades institucionales y de su transformación en Universidad Tecnológica, contribuyó al conocimiento del perfil de los egresados del subsistema técnico y sus posibilidades de acceder a una educación superior. Abocados al estudio de la enseñanza técnica en el nivel primario y medio dependiente del Ministerio de Instrucción Pública y Justicia se distinguen los aportes de Spregelburd (1997), Navarro (2015) y Pronko (2009). La primera autora logró reconstruir el interjuego entre las políticas educacionales nacionales que dieron origen a las Misiones Monotécnicas y de Extensión Cultural y las particularidades y significados que asumió una en una localidad bonaerense. Navarro enmarcó la creación de dichas instituciones en el cuadro de la educación rural salteña. Pronko, por su parte, investigó las iniciativas de la Unión Industrial Argentina en relación con las Escuelas Industriales de la Nación. El período histórico posterior fue abordado por Pineau (1997b) al reconstruir las reformulaciones del vínculo educacióntrabajo entre 1955 y 1983 a través del estudio de las políticas de educación técnica, aunque no analizó en detalle el tercer gobierno peronista. No obstante, resulta interesante su aporte ya que advirtió que, tras la caída del gobierno justicialista en 1955, se logró afianzar nuevamente la relación educación técnica-trabajo industrial en 1980 cuando, paradójicamente, dicha actividad económica fue desalentada por las políticas de corte monetarista y militarista de la última dictadura militar.

Sobre el período 1973-1976 la historiografía de la educación ha sido prolífica en los últimos años en cuanto al estudio del ámbito universitario (BESOKY, 2017; CARNAGUI Y ABBATISTA, 2004; FRIEDEMANN, 2016; 2017; DOVAL, 2001; IZAGUIRRE, 2011; RODRÍGUEZ, 2014; 2015) y, en menor medida, de la alfabetización de adultos y niños (MENDELA, 2015; TOSOLINI, 2010; PALACIOS Y RODRÍGUEZ, 2006; ABBATISTA, 2015).

Resultan de gran valor para este artículo otras investigaciones de Rodríguez (2013) y Abbattista (2013; 2019) puesto que abordaron las filiaciones ideológicas de los funcionarios de la cartera educativa. Con respecto a la problemática de la educación técnica nacional en el período 1973-1976 hay una evidente ausencia de estudios específicos.

El corpus de fuentes analizado en este artículo se compone en especial de documentos oficiales, entre los cuales se destacan: los discursos de los ministros de educación, ${ }^{3}$ los informes finales de las asambleas del Consejo Federal de Educación (CFE), el Boletín de Comunicaciones del Ministerio de Cultura y Educación (MCE), el Boletín del Consejo Nacional de Educación Técnica (CONET), el Boletín del Centro Nacional de Documentación e Información Educativa (CENDIE) y el Boletín Oficial de la República Argentina (BO). Estas fuentes fueron complementadas con los diarios Clarín y La Capital, de circulación nacional y regional respectivamente.

\section{La formación técnica en los proyectos de reformas educacionales}

El 25 de mayo de 1973, Héctor Cámpora asumió la presidencia de la Nación. Anteriormente se había desempeñado como diputado y secretario de Perón (BOETTO, 2015); durante su corto gobierno nombró como ministro de Cultura y Educación a Jorge Alberto Taiana, médico personal y del círculo de confianza de Perón. El Ministerio se conformó, entre otros, por jóvenes intelectuales militantes de la Tendencia Revolucionaria

\footnotetext{
${ }^{3}$ Se analizaron cinco discursos de Taiana, más de una decena de Ivanissevich, y tres de Arrighi.
} 
del Peronismo. Aunque esta línea no fue hegemónica al interior del Ministerio, dejó una impronta contundente en proyectos y programas al incorporar elementos de las políticas culturales y educacionales de la Cuba revolucionaria, del Movimiento de Izquierda Democrática Allendista de Chile y de la producción de varias generaciones de intelectuales argentinos de izquierda (ABBATTISTA, 2013; 2019).

En el mensaje inaugural de la Asamblea Legislativa de 1973 Cámpora atribuyó a la crisis del sistema educativo la "totalidad de los profundos problemas" que afectaban al país. Postulaba necesaria una revolución educativa y cultural con sentido nacional para superar la incongruencia entre las instituciones educacionales y los requerimientos de la Nación. La dependencia y la colonización económica debían ser combatidas en diversos campos, incluyendo al educacional. Sostenía que la escuela, para "servir al país," debía crear mentalidades independientes y consideraba imprescindible sancionar una ley nacional de educación para la cual debían habilitarse canales de consulta y participación de padres, docentes y estudiantes. Siendo la educación un patrimonio de la cultura popular, "el pueblo" debía participar en la definición de los objetivos del sistema educativo y asumir cierta la responsabilidad en su funcionamiento. Así, con sentido popular y cristiano, la escuela ajustaría su cometido a los requerimientos concretos del desarrollo social y económico del país. Esta ley debía trazar los lineamientos nacionales, generales y comunes que se complementarían con planificaciones regionalizadas y administraciones descentralizadas del sistema. ${ }^{4}$

Las pautas y concepciones educativas sostenidas por Cámpora fueron avaladas por los funcionarios de la cartera educativa. Entre sus fundamentos estaba la importancia de superar la segmentación que dividía los niveles y las orientaciones que escindían trabajo manual e intelectual en la enseñanza media, dado que esto se reflejaba en la elección prematura de una modalidad de enseñanza secundaria, desconociendo las demás ramas del saber. Las diferentes orientaciones de este nivel, agudizaban la preeminencia de la educación intelectual por sobre la técnica, desvalorizada por asociarse a tareas manuales y artesanales. Además, postulaban la importancia de institucionalizar la educación permanente, para atender a las exigencias de las innovaciones productivas, y de reconocer a la educación primaria y media como un bloque de formación inicial. La modernización didáctica, curricular y organizativa proyectada fue asociada con la participación social, palanca de una "educación popular." Proyectaban que las demandas educativas de las comunidades se relacionarían directamente con la satisfacción de las necesidades ocupacionales regionales, siendo necesaria una ampliación y diversificación de la oferta educacional, integrando conocimientos técnicos y humanísticos. El vínculo educacióntrabajo propuesto por el presidente Cámpora era avalado por Perón -referente clave de los gobernantes $\mathrm{y}$ funcionarios en ejercicio del poder-. El líder exiliado consideraba fundamental proveer capacitación laboral, no sólo por sus efectos en el trabajo productivo, sino por sus beneficios materiales y espirituales. ${ }^{5}$

En correspondencia con estos principios el ministro de Cultura y Educación, Jorge Taiana, exponía la importancia de la participación de la comunidad educativa, en forma ordenada y disciplinada, en la definición de la política educativa. Ello formaba parte del proceso más amplio de reconstrucción y liberación nacional, que permitía abrir la escuela al pueblo convirtiéndola en un centro de cultura popular y en un organismo proveedor "de los elementos

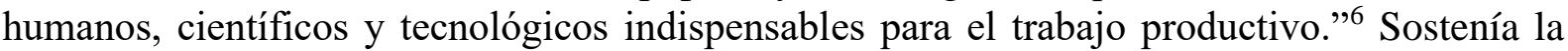
necesidad de explorar las vocaciones de los alumnos a lo largo de toda la enseñanza secundaria mostrándoles que la educación universitaria no era la única opción a seguir, sino que existía un

\footnotetext{
${ }^{4}$ Congreso de la Nación, Mensaje del Presidente de la Nación Argentina Dr. Héctor José Cámpora al inaugurar el $98^{\circ}$ período ordinario de sesiones del Honorable Congreso Nacional. Bs. As.: Imprenta del Congreso de la Nación, 1973.

${ }^{5}$ TAIANA, Jorge, "Cultura popular", Boletín de Comunicaciones $\mathrm{N}^{\circ} 3$. XVI. 15/6/1973.

6 "La educación debe tener una salida laboral, afirmó Taiana", Clarín, 5/12/1973, p. 17.
} 
abanico diversificado de actividades y profesiones entre las cuales podían elegir y aportar al desarrollo socio-económico. El ministro defendía una formación que superase las antinomias entre trabajo manual e intelectual y que ofreciese una salida laboral a los egresados para evitar una formación innecesariamente academicista. ${ }^{7}$

Pese a la sucesión presidencial de Cámpora por Lastiri -y de éste último por Perón- y al desplazamiento en el ministerio de aquellos jóvenes de la Tendencia Revolucionaria del Peronismo (ABBATTISTA, 2013), los principios educativos que propusieron fueron incluidos en las recomendaciones formuladas en las Asambleas del CFE de septiembre y noviembre de 1973. En ellas se comenzó a trabajar en la sanción de una ley nacional de educación. Se establecieron las Pautas Programáticas de la Política Educativa Argentina que ratificaron la importancia de la participación social en la educación y definieron la finalidad de la educación. Para alcanzar el cometido de liberación, la educación debía reivindicar los valores nacionales y populares -fundamentalmente la justicia social y el antiimperialismo-y formar "críticamente" para la producción y la conducción. Se recomendó, entre otras cuestiones, las siguientes acciones: enseñar labores técnicas en los últimos años de la enseñanza primaria y en el nivel medio; conocer las expectativas sociales sobre éste ciclo para responder a demandas concretas; descentralizar la conducción técnica y pedagógica; reemplazar el calendario académico único por uno que respetase y contemplase las particularidades regionales y locales; facilitar el reconocimiento de títulos y equivalencias de estudio.

Con relación a la educación técnica, en esas asambleas del CFE se trataron también los problemas que planteaban las diferentes incumbencias de los títulos emitidos por escuelas técnicas de distintas jurisdicciones y se consensuó la necesidad de reformar el impuesto a la educación técnica. ${ }^{8}$ Los funcionarios reunidos en las sesiones del CFE consideraban necesario otorgar coparticipación del tributo a las provincias que sostenían escuelas técnicas y otorgarle potestad al CONET para decidir sobre la creación de establecimientos técnicos privados, suprimiendo la facultad de las empresas de decidir cuáles cursos y escuelas abrían y subvencionaban. ${ }^{9}$

A pocos meses del inicio de la presidencia de Perón fue publicado el Plan Trienal para la Reconstrucción y la Liberación Nacional 1974-1977 a través del cual apoyó la gestión ministerial de Taiana. Pese a la hostilidad del clima político y a los numerosos desencuentros entre el Presidente y las organizaciones en las que militaban funcionarios que permanecían en la cartera educativa, el Poder Ejecutivo aprobó las acciones emprendidas (ABBATTISTA, 2013). En el Plan Trienal se retomaron principios explicitados por Cámpora y Taiana y las recomendaciones de las Asambleas del CFE. La educación fue concebida como un "proceso de alistamiento de nuevas y postergadas categorías sociales" para que se incorporasen "al trabajo efectivo y a los poderes de decisión" y presentada como una "inversión rentable" en el marco de un programa económico nacional destinado a la liberación. Se estableció una lista de prioridades encabezada por la erradicación del analfabetismo, la disminución del semianalfabetismo, la estimulación de la educación permanente y la expansión de los jardines de infantes. Luego se ubicaba como prioridad a la capacitación técnica, que debía ser transversal tanto en el nivel primario como el secundario, disolviendo las fronteras artificiales entre educación teórica y práctica, y debía tender a hacer del "estudiante un trabajador y del trabajador un estudiante". La enseñanza se debía adaptar a las peculiaridades geográficas,

\footnotetext{
${ }^{7}$ CFE, III Reunión ordinaria de la Asamblea General. Informe final. Anexos. Serie legislación educativa No 4. Bs. As.: CENDIE, 1973.

${ }^{8}$ El impuesto para aprendizaje cambió su denominación en 1962, convirtiéndose en impuesto para la educación técnica.

${ }^{9}$ CFE, III Reunión ordinaria de la Asamblea General. Informe final. Anexos. Bs. As., 1973. CFE, “Anexo 9. Índice.” En Asamblea extraordinaria del CFE. Chaco. 4-5/9/1973. Bs. As., 1973.
} 
culturales, históricas y socioeconómicas de las diversas regiones para vincularse con el trabajo productivo. Para que se cumplieran los objetivos propuestos se proyectaba emprender obras de infraestructura y descentralizar el Ministerio de Educación, creando delegaciones regionales de asistencia técnica-docente. ${ }^{10}$

En concordancia con esa planificación, el ministro Taiana consideraba que el déficit más agudo del sistema educativo estaba en el nivel medio. Enfatizaba la necesidad de reorientarlo para que formase jóvenes capaces de incrementar la fuerza laboral y de asumir una función protagónica en el proceso de reconstrucción nacional. ${ }^{11}$ Respecto a la educación técnica sostenía que debía actualizarse con tecnología moderna e incluir contenidos humanistas, a la vez que tenían que acotarse las carreras, adecuándolas a las múltiples salidas laborales. ${ }^{12}$

El Subsecretario de Educación de la Nación, Reynaldo Ocerín, explicaba que para alcanzar el cometido del Plan Trienal en relación al nivel medio era necesario lograr su universalización y modernización. Se proyectaba ampliar la cantidad de establecimientos así como la matrícula; incrementar el número de escuelas polivalentes ${ }^{13} \mathrm{y}$ vincular el nivel medio con la educación permanente. Para modernizarlo era necesario reformular los contenidos culturales -no profesionales-; implementar un ciclo básico común; proveer salidas laborales mediante la expansión de las modalidades de educación agrotécnica, técnica-comercial, artística y la transformación del bachillerato. ${ }^{14}$

En abril de 1974, en la IV Reunión ordinaria de la Asamblea General del CFE, se aprobó el despacho de la Comisión de Legislación Educativa de Enseñanza Media. El mismo proponía replantear todos los aspectos funcionales y operativos del nivel: el gobierno de la educación, los estatutos, leyes y reglamentos; las modalidades de evaluación y promoción, para asegurar mayor retención de los alumnos; la formación docente, para que adquiera impronta regional; y la infraestructura edilicia y la denominación de las unidades escolares, para unificarlas. Fuera de discusión estaban la extensión de la obligatoriedad y el cambio en las formas de enseñanza, ambas aprobadas en esa instancia. En la enseñanza media general se proponía la obligatoriedad de un nuevo ciclo básico común de tres años que debía propiciar el desarrollo armónico y equilibrado de los adolescentes y

facilitar la adquisición de habilidades básicas para el ejercicio futuro de profesiones y oficios; [...] lograr una preparación que torne a los educandos en sujetos aptos para ingresar, eventualmente, al proceso de producción [...] introducir el trabajo por áreas programáticas para superar los males del enciclopedismo y de la mera yuxtaposición de conocimientos, y al mismo tiempo, trabajar con los grandes principios organizadores de los distintos contenidos. ${ }^{15}$

Las resoluciones del CFE indicaban que en el ciclo básico se debían desarrollar aspectos espirituales y procedimentales: estimular el nacionalismo, la solidaridad, el orden, el valor práctico del trabajo manual y técnico y artístico, entre otros; orientar vocacional y profesionalmente al alumno y capacitarlo para el eficiente desempeño en el

\footnotetext{
${ }^{10}$ MCE, Plan Trienal 1974-1977. Bs. As.: CENDIE, 1974, p. 10.

11 "Retorno la actividad a las escuelas al iniciarse ayer el nuevo ciclo lectivo", Clarín, 19/3/1974, p. 48.

${ }^{12} \mathrm{MCE}$, Discurso pronunciado por el Sr. Ministro Jorge Alberto Taiana al iniciarse el ciclo lectivo 1974. 18 de marzo. Concepción del Uruguay. Entre Ríos. Bs. As., 1974.

${ }^{13}$ Las escuelas medias polivalentes, creadas por el MCE desde 1970, proporcionaban distintas modalidades de educación media con una mínima inversión ya que hacían uso de los talleres de las escuelas técnicas y/o de instalaciones de organizaciones estatales y privadas para la realización de las prácticas de agronomía.

${ }^{14}$ MCE, Boletín de Comunicaciones $\mathrm{N}^{\mathrm{o}}$ 10-11. XVI. Sin fecha (s/f), p. 11.

15 MCE, "Coparticipación en un impuesto. Impuesto a la educación técnica", Boletín de Comunicaciones $\mathrm{N}^{\circ}$ 8-9. XVI. S/f, p. 6.
} 
mundo del trabajo y en el nivel superior. Alentaban cambios en las metodologías de enseñanza: serían creadas áreas en función de los principios organizadores de los distintos conocimientos, integrando contenidos mínimos institucionales, particularidades regionales y preferencias de los alumnos. ${ }^{16}$

\section{El conet frente a los programas de cambio educativo radical}

En sintonía con las intenciones de Cámpora, Taiana y los funcionarios del Ministerio de educación en cuanto a vincular educación y trabajo, el presidente del CONET, ingeniero Carlos Benítez, consideraba que la educación técnica debía estimularse por su impacto en la industria. Pensaba que la formación de técnicos nacionales era clave para lograr la independencia económica, cambiar el rumbo del comercio internacional y reposicionar al país en el concierto de las naciones. La completa reorganización del subsistema de enseñanza técnica aumentaría el bienestar general por lo que dichas acciones se harían en nombre del pueblo, protagonista principal del movimiento de liberación. Dadas las características de esta modalidad de enseñanza, era una prioridad contar equipamiento actualizado para garantizar la práctica eficaz de cada alumno. ${ }^{17}$

Durante la segunda mitad de 1973, cuando la presidencia de la Nación pasó de Cámpora a Lastiri y luego a Perón, en las escuelas técnicas nacionales se auspició la creación experimental de instancias que permitían la participación de la comunidad en el gobierno y en la administración de la educación, dado que se constituyeron centros de asesores de padres. Debían facilitar el seguimiento estrecho de los alumnos y colaborar en el alcance de los objetivos educativos. ${ }^{18}$ También los directivos, maestros de enseñanza práctica y alumnos integraron mesas de trabajo en las que debatieron los planes de estudio. Con el propósito de regionalizar la enseñanza se le dio participación a entidades gremiales empresarias en el debate sobre la actualización curricular. Gobernantes provinciales y municipales gestionaron con funcionarios del CONET proyectos tendientes a mejorar y ampliar los alcances de la educación técnica en zonas periféricas y a lograr el medio boleto de trasporte urbano para los alumnos. ${ }^{19}$

En septiembre de 1973 se llevaron a cabo acciones que promovían la autarquía y autonomía del CONET. El gobierno peronista derogó la legislación que había descentralizado a este en un organismo en lo referido a la administración y conducción operativa de las unidades escolares. ${ }^{20}$ De este modo se le otorgaron garantías para su pleno funcionamiento, eliminando las políticas limitaciones impuestas durante la autodenominada "Revolución Argentina". ${ }^{21}$

Tras la publicación del Plan Trienal para la Reconstrucción y la Liberación Nacional, en diciembre de 1973, el Presidente del CONET ratificó sus ideas acerca de que la soberanía y la independencia debían ser sustentadas por una industria y tecnología nacional altamente desarrollada, puesto que una economía exclusivamente agropecuaria sería incapaz de convertir al país en una potencia. En consecuencia, atribuía gran gravitación a la educación

\footnotetext{
${ }^{16}$ CFE, IV Reunión ordinaria de la Asamblea General. Informe final. Serie legislación educativa $\mathrm{N}^{\mathrm{o}} 7$. Bs. As.: CENDIE, 1974.

${ }^{17}$ BENÍTEZ, Carlos, “Al personal y a los alumnos del CONET”, Boletín del CONET No 386. 25/6/1973, pp. 597-598. MCE, "La participación del pueblo como instrumento del cambio educativo", Boletín de Comunicaciones $\mathrm{N}^{\circ}$ 3. XVI. 15/6/1973, p. 11. CONET, La educación técnica en el logro de los objetivos para la reconstrucción y liberación nacional. Cursos Sindicales. Bs. As., 1974.

${ }^{18}$ CONET, "Disposición DGP No 160. 16/7/1973. ENET No 1. Participación de los alumnos en actividades escolares. Creación de centros de asesores de padres de alumnos en escuelas técnicas”, Boletín del CONET No 391. 6/8/1973, p. 767.

19 "La ubicación de una nueva escuela técnica es motivo de estudios", La Capital, 4/9/1973, p. 3. "El presidente del CONET reuniráse con empresarios", La Capital, 8/8/1973, p. 6. "Interesante coordinación para expandir la enseñanza técnica: una nueva escuela”, La Capital, 10/8/1973, p. 5.

${ }^{20}$ B.O. "Se derogan las llamadas leyes $20.014,20.015$ y 20.016. Ley 20.527."

${ }^{21}$ B.O. "Ley No 20.016/72. Art. 2." Año LXXXI. No 22.582. Bs. As., 10/1/73, p. 5.
} 
técnica y al CONET, proponiéndose formar 30.000 técnicos, 70.000 operarios calificados y 650.000 operarios con un mínimo de calificación, atendiendo a la diversidad económicoproductiva del país. ${ }^{22}$ Para alcanzar dichas cifras y propósitos se amplió la oferta a través de la creación nuevas escuelas y del fortalecimiento de las instancias de capacitación laboral, perfeccionamiento y reconversión profesional. Además propició transformar cualitativamente a la oferta y a la dinámica de enseñanza en el nivel medio. La estructura de ese nivel, integrada por el ciclo básico y superior, que otorgaba dos titulaciones alternativas (Auxiliar Técnico y Técnico) fue objetada por los funcionarios del CONET, porque consideraban que los problemas socioeconómicos dificultaban el sostenimiento del cursado en doble turno y no existía una correspondencia real entre la formación adquirida en cada ciclo y las demandas efectivas del mercado de trabajo.

En el documento "La educación técnica en el logro de los objetivos para la reconstrucción y liberación nacional," ${ }^{23}$ publicado en ese momento, se planteaba que la evolución tecnológica no era un fin en sí mismo sino que su meta era la contribución a la felicidad y libertad del pueblo: se trataba de un objetivo solidario, tendiente a la constitución de modelos de producción y de consumo propios, que respondiesen a las necesidades de la sociedad argentina. La capacitación de la mano de obra, entonces, debía ser atendida con carácter prioritario por el gobierno y los empresarios. Se trataba tanto de una necesidad del Estado como de un derecho del hombre, inherente a su papel de protagonista del quehacer económico nacional. En este sentido, la formación no era concebida sólo como la transmisión de conocimientos que habilitaban para el desempeño en una actividad económica sino que se proyectaba como parte de una formación integral que entendía al trabajador como "ser humano, partícipe de la sociedad". ${ }^{24}$

Según se postulaba, la educación integral permitiría al alumnado adquirir y avanzar en los niveles de decisión y elección necesarios para contribuir al desarrollo de tecnología y garantizaría la formación de mano de obra ajustada a las necesidades ocupacionales. Concretamente, se propuso reestructurar el plan de estudio de las ENETs. El nuevo diseño curricular constaba de tres etapas de dos años cada una, que se correspondían con distintos grados de acercamiento al medio laboral y habilitaban diversas salidas de empleo. La primera era un ciclo básico común tendiente la formación integral de los estudiantes, que proporcionaba conocimientos culturales y profesionales y ofreciendo experiencias en diversas actividades laborales y habilitaba para continuar estudios superiores e iniciarse en distintos puestos de trabajo. En el siguiente ciclo, que correspondía al de auxiliares técnicos, se enfatizaría en la cultura profesional con aplicación práctica y proveería a los alumnos una capacitación profesional relacionada con una rama de la actividad productiva. El objetivo del ciclo técnico superior era preparar para la conducción y la investigación en una especialidad determinada. El plan se ajustaba a las premisas de la educación permanente y de la demanda de la actividad industrial. Se esperaba que la enseñanza escalonada contribuyese a superar los problemas de deserción y marginalidad, a consolidar el "ser nacional" y a lograr efectiva gravitación sobre la producción.

Además, a través del programa de acción derivado del Plan Trienal se proyectó rehabilitar a las escuelas-fábricas e instalar ciclos de formación profesional para adolescentes. Según argumentaban los funcionarios del CONET, aseguraban congruencia y complementariedad entre los requerimientos de la industria y la formación de la mano de obra,

22 MCE, "Presidente del Consejo Nacional de Educación Técnica Carlos A. Benítez", Boletín de Comunicaciones $\mathrm{N}^{\circ}$ 10-11. XVI. S/f, p. 19.

${ }^{23}$ CONET, La educación técnica en el logro de los objetivos para la reconstrucción y liberación nacional. Cursos Sindicales. Bs. As., 1974.

${ }^{24}$ CONET, La educación técnica en el logro de los objetivos para la reconstrucción y liberación nacional. Cursos Sindicales. Bs. As., 1974, p. 56. Las cursivas son nuestras. 
por lo que se constituyó una comisión para restablecerlas. ${ }^{25}$ Los ciclos de formación profesional para adolescentes estarían orientados a jóvenes de más de 14 años que habían aprobado cuanto menos el quinto grado de la escolaridad primaria -o bien habían abandonado la educación media- y se ubicarían en núcleos urbanos con plantas industriales.

Estas políticas de educación técnica ratifican la afirmación de Abbattista (2013) al respecto de que la política cultural de los funcionarios del Ministerio de Educación afines a la Tendencia Revolucionaria del Peronismo mantuvieron los lineamientos de las políticas de los primeros gobiernos justicialistas en cuanto a la educación técnica. Los cambios operados en las propuestas de enseñanza del CONET, sin embargo, no alcanzaron la envergadura de las transformaciones propuestas en el Plan Trienal. Con la progresiva expulsión de los funcionarios que acompañaban a Taiana ocurrida durante 1974, se mantuvieron los planes de estudios vigentes. Las únicas modificaciones registradas en el currículo del nivel medio fueron aquellas vinculadas al reemplazo de la asignatura Educación Democrática por Estudio de la Realidad Social Argentina (ERSA) y a la actualización de los ciclos superiores de las especialidades Electromecánica y Administración de empresas. ${ }^{26}$

Las iniciativas que progresaron fueron aquellas relacionadas con la aprobación del plan de estudios del Ciclo de Formación Profesional para Adolescentes, de dos años de duración, que habilitaba para acceder a puestos de trabajo determinados, acordes con las edades. El plan estaba integrado por tres áreas y se componía de actividades obligatorias y optativas. Entre las primeras se impartía un área de cultura general que tenía como objetivo complementar la enseñanza básica; comprendía, entre otras asignaturas, Legislación del Trabajo y ERSA. El área de cultura profesional también era obligatoria; tendía al desarrollo de habilidades manuales y a brindar la "polivalencia" requerida para la adaptación al mercado laboral y constaba de las materias Dibujo técnico y Práctica de taller. Las actividades complementarias o de recuperación, eran educación física y recreación. ${ }^{27}$ Las especialidades en las que formaban estos Ciclos eran: Albañilería, Artes Gráficas, Industria de la alimentación, Industria del vestido y afines, Instalaciones domiciliarias, Industrias químicas, Mecánica automotriz, Mecánica general, Metalurgia, Minería, Tecnología agropecuaria, Tecnología textil y Telecomunicaciones. ${ }^{28}$ Para su implementación se crearon catorce ENETs, denominadas centros de formación para adolescentes, a través de convenios con gobernaciones y reparticiones oficiales.

\section{La derecha peronista en el poder y el cambio de agenda educativa}

Tras la muerte del presidente Perón, ocurrida el primero de julio de 1974, hubo cambios en el gabinete nacional. En un clima de creciente conflictividad social y violencia política, María Estela Martínez asumió la presidencia de la Nación y reemplazó al ministro Jorge Taiana por Oscar Ivanissevich. El gobierno fortaleció la alianza de la derecha peronista con las Fuerzas Armadas y los capitalistas más concentrados, sustituyendo a los funcionarios políticos que representaban a la burguesía nacional y a los "moderados" por hombres de derecha (SERVETTO Y PAIARO, 2013; ROUGIER Y FISZBEIN, 2006). En el MCE fueron convocados muchos de los funcionarios de la cartera educativa de los

\footnotetext{
${ }^{25}$ CONET, Resolución No 266. 28/2/1974, Boletín del CONET No 414. 11/3/74, p. 74.

${ }^{26}$ CONET, Resolución No 1184. 11/7/1973, Boletín del CONET No 391. 6/8/73, p. 56; CONET, Resolución $\mathrm{N}^{\mathrm{o}}$ 370. 13/3/1974, Boletín del CONET No 418. 26/3/74, pp. 166-168. CONET, Resolución No 150. 5/2/1975, Boletín del CONET No 469. 3/3/1976, pp. 32-33.

27 "Reordenamiento educativo. Nota editorial”, Clarín, 1/3/1974, p. 8; CONET, Resolución N 266. 28/2/1974, Boletín del CONET No 414. 11/3/74, p. 74; CONET, Resolución No 1343. 25/7/1974, Boletín del CONET No 440. 5/8/74, p. 685.

${ }^{28}$ CONET, La educación técnica en el logro de los objetivos para la reconstrucción y liberación nacional. Cursos Sindicales. Bs. As., 1974. MCE, "Nuevo plan de estudio del CONET", Boletín del CENDIE N 14. Julio-septiembre 1974.
} 
primeros gobiernos justicialistas -Carlos Frattini, Pedro Andrés Saggese, entre otros(ABBATTISTA, 2013; 2019). María Estela Martínez postulaba que el propósito de la educación era lograr la identificación del alma y la vida de los alumnos con el territorio argentino y su tradición espiritual, para afirmar el equilibrio armónico entre el individuo y la sociedad, consolidando la comunidad organizada. ${ }^{29}$

El ministro Ivanissevich se presentaba como un devoto adherente al nacionalismo cristiano y justicialista, que había ayudado a constituir junto a Perón cuando se desempeñó como ministro de Educación desde 1948 a 1950. Consideraba que su nombramiento se vinculaba con la necesidad de defender la doctrina y orientar al pueblo en una salida nacional, frente al estado "convulsivo y revolucionario" que primaba. ${ }^{30}$ Según anunció días más tarde de su asunción como ministro, la prioridad de la República era la educación y la misión del Ministerio era el "rescate espiritual del país" que implicaba la restitución de valores justicialistas: el cristianismo, la familia, la justicia, la disciplina, la independencia y la soberanía, dado que la escuela argentina se había perdido en "un internacionalismo materialista." La desaparición del ser nacional, para el ministro, era mucho más grave que la de la tierra y la economía. Sostenía que "salvar el alma de la Patria" era un requisito para recuperar todo lo demás. ${ }^{31}$ Antes que formar productores y conductores, las razones de ser de la escuela y de la educación eran la transformación nacionalista y cristiana de los "zurdos", el control y la humanización de las reacciones animales y de los "instintos primos"; el perfeccionamiento de la "persona humana" para que fuese capaz de trascender y convivir en una comunidad organizada basada en la tradición argentina. ${ }^{32}$ El ministro afirmaba que el desarrollo humano se conquistaba con educación, que permitía la convivencia y la instrucción. Consideraba que la primera debía conducir a que surgiese del hombre su origen divino. Las profesiones universitarias liberales, según su opinión, formaban egresados frustrados por la falta de empleo. Consideraba necesario impulsar una política de formación de recursos humanos a través de la creación de "centros de educación superior" en el nivel terciario y la estimulación de las carreras universitarias técnicas. ${ }^{33}$

Para Ivanisevich, la pérdida de eficacia de la institución escolar se debía a que había sido dirigida por personas cuyas tendencias eran contrarias a la Doctrina Nacional y había sido consecuencia de las concesiones laborales reconocidas por el Estatuto Docente de $1958 .{ }^{34}$ El trabajo docente, para el ministro, se constituía de tres tareas: la formación moral requerida para convertir "una semilla en un ser humano;" la de ser ejemplo moral y ético de sus alumnos; y la de compartir y complementar las acciones educativas de las familias. ${ }^{35}$ Postulaba que la segunda obligación, que ponía a los docentes en el lugar de modelos, era

${ }^{29}$ CONET, "Palabras de la excelentísima Sra. presidente de la Nación doña María Estela Martínez de Perón, en el día de la iniciación del período lectivo de 1975", Boletín CONET No 478. 14/4/1975, pp. 271-275.

30 “Juraron ayer los nuevos ministros del Interior, Defensa y Cultura y Educación", Clarín, 15/8/1974, pp. 16-17.

${ }^{31} \mathrm{MCE}$, "El señor ministro de cultura y educación Dr. Oscar Ivanissevich habló a los docentes y al país con motivo del día del maestro", Boletín del CENDIE No 14. Julio-septiembre 1974.

${ }^{32} \mathrm{MCE}$, "El señor Ministro de Cultura y Educación Dr. Oscar Ivanissevich habló a los docentes y al país con motivo del día del maestro", Boletín del CENDIE No 14. Julio-septiembre 1974. MCE, Discurso pronunciado por el Ministro de Cultura y Educación Dr. Oscar Ivanissevich en el acto realizado en celebración del $158^{\circ}$ aniversario de la batalla de Chacabuco. 12/2/1975. Bs. As., 1975.

33 “Capacitación y salida laboral. Nota editorial”, Clarín, 2/10/1974, p. 8. MCE, Mensaje de S.E. el Sr. Ministro de Cultura y Educación Dr. Oscar Ivanissevich, a los Ministros de cultura y educación de las provincias argentinas y a los rectores e interventores de las Universidades Nacionales. 26/7/1975. Bs. As., 1975.

${ }^{34} \mathrm{MCE}$, Mensaje de su Excelencia el señor Ministro de Cultura y Educación doctor Oscar Ivanissevich. 10 de setiembre de 1974. Centro Nacional de documentación e información educativa. Bs. As., 1974.

${ }^{35} \mathrm{MCE}$, Mensaje dirigido a los maestros por el Ministro de Cultura y Educación, Dr. Oscar Ivanissevich el 3 de septiembre de 1974, debido a la repetición de medidas de fuerza que afectan a la escuelas y a la educación. Bs. As., 1974. 
de vital importancia porque "la didáctica" era "el ejemplo". ${ }^{36}$ Fundamentado en dichas premisas, el ministro cuestionó en reiteradas ocasiones el desempeño docente. Consideraba que si bien el derecho a huelga existía era contrario a los principios pedagógicos en los que se fundaba la docencia ${ }^{37} \mathrm{y}$, dadas las circunstancias y los esfuerzos estatales para recomponer los salarios, la concebía extemporánea e ilegal. ${ }^{38}$

En noviembre de 1974 se declaró Estado de Sitio por lo que se adelantó la finalización del ciclo lectivo en muchas escuelas por los "actos subversivos" que habrían cometido sus alumnos. Policías y militares custodiaban establecimientos de Capital Federal y Buenos Aires; el gremio CTERA dejó de ser considerado como núcleo de agremiación docente y, en su reemplazo, se asignó personería jurídica a la Unión de Docentes Argentinos (ABBATTISTA, 2019).

En mayo de 1975, el ministro consideraba en que no había logrado alcanzar el objetivo de su gestión: la argentinización de la escuela. ${ }^{39}$ En ese momento, manifestó su intención seguir los lineamientos del Plan Trienal pese a oponerse a la regionalización de la enseñanza y a la descentralización administrativa del ministerio, lineamientos centrales del documento. ${ }^{40}$

En los meses de junio y julio de ese año representantes de la Universidad Nacional y los ministros de todas las provincias fueron convocados para "homogeneizar la conducción" y considerar el anteproyecto de la Ley Fundamental de la Educación Argentina (ABBATTISTA, 2019). Sin embargo, no lograron consensos al respecto. Los representantes provinciales se pronunciaron en favor de la recomposición de los salarios docentes ${ }^{41}$ al tiempo que Ivanissevich ratificó su interés más centrado en el disciplinamiento y la censura que en cuestiones propositivas vinculadas a conocimientos teóricos y prácticos.

A fines de 1974, en un marco de indefinición de políticas educacionales y de alta conflictividad político estudiantil, desde el CONET comenzaron a promoverse salidas y cambios alternativos a los diseñados durante la gestión de Taiana. Los proyectos que, según los funcionarios del CONET, permitían combinar elementos de una educación integral con las demandas del mercado de empleo fueron desconsiderados por el gabinete de Ivanissevich. De modo que el proyecto que buscó rehabilitar el formato institucional de las escuelas-fábricas también fue desestimado. Las alternativas que encontraron en 1975 para crear modalidades de formación acordes con algunos de los fundamentos que sostenía sobre la educación técnica retomaron estrategias ya aplicadas en el organismo.

En el nivel medio el rechazo presentado por las nuevas autoridades ministeriales al reemplazo de la estructura curricular de dos ciclos de tres años, por una novedosa, de tres ciclos de dos años de duración cada uno, condujo a que, como paliativo, se impusiesen con carácter obligatorio los cursos de Auxiliares Técnicos que exigían a los egresados del ciclo básico el cursado del "cuarto año de término". Según las autoridades del CONET muchos alumnos abandonaban sus estudios tras concluir el ciclo básico y era evidente la carencia de mano de obra formada en el nivel de "ejecución", que podía ser satisfecha con

\footnotetext{
${ }^{36} \mathrm{MCE}$, Disertación presentada por el Sr. Ministro de Cultura y Educación de la nación, Dr. O. Ivanissevich, refiriéndose al enfoque Gral. del sistema educativo (25/2/1975). Bs. As., 1975

${ }^{37} \mathrm{MCE}$, "El Ministro de Cultura y Educación habló sobre las huelgas docentes (17/6/1975)". En Comunicado de prensa $N^{o} 174$. Bs. As., 1975.

${ }^{38}$ MCE, Mensaje dirigido a los maestros por el Ministro de Cultura y Educación, Dr. Oscar Ivanissevich el 3 de septiembre de 1974, debido a la repetición de medidas de fuerza que afectan a la escuelas y a la educación. Bs. As., 1974.

${ }^{39} \mathrm{MCE}$, Sr. Ministro de Cultura y Educación Dr. Oscar Ivanissevich. Mensaje al Congreso Nacional. 1/5/1975. Bs. As., 1975.

${ }^{40}$ MCE, "Disertación del señor Ministro de Cultura y Educación de la Nación, doctor Oscar Ivanissevich, refiriéndose al enfoque general del sistema educativo nacional", Boletín del CENDIE No 16. Enero-marzo 1975.

${ }^{41}$ MCE, Declaración de los ministros de educación de las provincias Argentinas. 26/7/1975. Bs. As., 1975.
} 
el aumento de los egresos de esos auxiliares. ${ }^{42}$ Con este propósito se ordenó a las direcciones de las ENETs que debían difundirlos y solicitar su apertura en el caso de no disponerlos. Además se dispuso que debían elaborar los programas de las asignaturas de Tecnología de la especialidad y del Taller del oficio del curso de término, considerando la infraestructura escolar, las normas didácticas y las necesidades del mercado educacionales la zona. ${ }^{43}$ La oferta de estos cursos se amplió en forma significativa durante 1975. Por otra parte, se dispuso un régimen de excepción que permitía a los alumnos del Ciclo Superior realizar las actividades prácticas en unidades industriales estatales, mixtas o privadas y se habilitó un Servicio de Orientación Vocacional. El régimen de excepción mencionado permitía a los alumnos que trabajaban en rubros afines a la especialidad cursada la promoción de las asignaturas Taller o Trabajos Prácticos, que se dictaba en contra turno. De este modo, hacía posible mantener los estudios y el empleo, ya que no requería la asistencia a las clases prácticas. El nuevo sistema favorecía la formación de un perfil profesional técnico más apropiado y una mejor comprensión del mundo laboral asegurando un aporte de los recursos humanos según las necesidades de las regiones económicas-productivas. El alumno tenía un seguimiento de sus labores en la empresa por parte de las autoridades escolares, obteniendo una calificación anual. ${ }^{44} \mathrm{~A}$ mediados de 1975, el CONET, incorporó un Servicio de Asistencia y Orientación Vocacional. La orientación vocacional era reconocida como una de las principales facetas de la educación y se aspiraba a implementarla gradualmente alcanzando a todos los estudiantes, para facilitar el proceso de enseñanza-aprendizaje, superar las dificultades que presentaba el pasaje de la escuela primaria al Ciclo Básico y aportar elementos que permitieran al alumno hacer elecciones más adecuadas. ${ }^{45}$ Se reconocía que su implementación implicaba un esfuerzo económico considerable para el CONET pero que el mismo era inevitable.

Tras un año de gestión y desanimado por los relativos avances de la educación espiritualista y nacionalista que sostenía, el ministro Oscar Ivanissevich renunció alegando problemas de salud. Lo sucedió en el cargo Pedro José Arrighi. El nuevo ministro se había desempeñado como interventor de la Universidad Provincial de Mar del Plata y de la Universidad Nacional de La Plata desde la que, según las acusaciones estudiantiles, había ejercido represión indiscriminada. Se mostraba "profundamente católico" y amigo de su predecesor en la cartera educacional (RODRÍGUEZ, 2014; 2015). Concebía que la labor docente era preparar a los hombres del mañana para sus encuentros con el destino trascendente y, en la coyuntura específica que atravesaban, hacer justicia por los muertos víctimas de la guerrilla y la subversión. Solicitaba al magisterio serenidad, comprensión y capacidad de diálogo, dado que consideraba que las huelgas no solucionaban los problemas que los afectaban. ${ }^{46}$

Además de esa acción contra la subversión, el ministro postulaba la necesidad de sancionar una ley de educación y de alcanzar los objetivos del Plan Trienal. No obstante, consideraba necesario evaluar con rigurosidad las experiencias y los proyectos derivados del mismo para ajustarlos, corregirlos, reorientarlos o eliminarlos. ${ }^{47}$ Las deficiencias de la

\footnotetext{
${ }^{42}$ CONET, Circular N ${ }^{\circ} 75$. "Promoción del Curso de término para formación de auxiliares técnicos", Boletín del CONET No 449. 23/9/1974, pp. 857-859.

${ }^{43}$ CONET, Resolución No 662. 15/4/1975. Boletín del CONET No 480. 28/4/1975, p. 366.

${ }^{44}$ CONET, Resolución No 167. 6/2/1975, Boletín del CONET No 471, pp. 49-53. CONET, Resolución Nº 25. 10/7/1975, Boletín del CONET No 494. 21/7/1975, 845.

${ }^{45}$ CONET, Circular No 133 , Boletín del CONET No 491. 7/7/1975, p. 753.

${ }^{46}$ ARRIGHI, Pedro. "La educación en el país. Discurso pronunciado por el Sr. Ministro de Cultura y Educación con motivo del día del maestro", Bs. As., 1975. MCE, "El Ministro, Dr. Pedro José Arrighi: La Educación contra la subversión", Boletín de Comunicaciones No 33-34. XVIII. 30/10/1975, p. 1. "El Ministro de cultura hizo declaraciones", La Capital, 5/10/1975, p. 3.

47 "Balance de realizaciones en el área Educacional”, Clarín, 20/12/1975, pp. 9 y 17. "Arrighi habló del sistema educativo", La Capital, 16/3/1976, p. 1.
} 
educación secundaria lo alarmaban, porque la percibía ineficaz para transmitir los valores cristianos y nacionalistas e imponer disciplina, lo cual la convertía en un "campo de reclutamiento y entrenamiento para la guerrilla." 48 Además, debía ser transformada para garantizar una formación de técnicos que colaborasen en el desarrollo de la riqueza nacional. Anunció que buscaría regular el ingreso de los alumnos para favorecer a los hogares de menos recursos y expandir el Proyecto 13, que venía implementándose desde 1970 y sostenía un cambio en el régimen laboral de profesores (en lugar de trabajar por horas, eran designados por cargo). ${ }^{49}$ Con respecto a la educación técnica aseguraba que, pese a las limitaciones de índole económica, pondría todo su empeño para que progresara, ${ }^{50}$ proponiéndose finalizar la construcción de ocho escuelas del CONET. ${ }^{51}$

\section{Conclusiones}

La heterogeneidad ideológica de los funcionarios del tercer peronismo se manifestó en los contrastes de sus discursos y perfiles. En ellos se condensaron sentidos y propuestas divergentes que ratifican la imposibilidad de concebir una imagen única del movimiento peronista. Tomando los perfiles de los gobernantes y funcionarios, los discursos y las agendas de cambio se reconocen dos momentos claramente diferenciados. A diferencia de los discursos de Cámpora y Taiana -en los que primaba una intención propositiva sobre cambios en las estructuras del gobierno del sistema y de la enseñanza-, los de Estela Martínez e Ivanissevich mantenían un perfil filosófico y espiritual, apelaban permanentemente a la doctrina moral y cristiana del peronismo y pretendían suscitar cambios en las conductas y en las conciencias de docentes y alumnos. En los discursos de Arrighi se combinaban ambos componentes: manifestaba intenciones vinculadas al cambio de conductas de la comunidad educativa, el espiritualismo, la justicia por los muertos por la guerrilla y algunas proyecciones de acciones concretas en el sistema.

Todos los gobernantes y ministros analizados concebían que el sistema educativo estaba en crisis, no obstante los factores críticos que enunciaban diferían. En el caso de Cámpora y Taiana, estaba vinculada a la dependencia y colonización económica, lo cual exigía afianzar el vínculo entre educación y trabajo en la educación media; la formación de recursos humanos apropiada para el desenvolvimiento eficaz en los medios productivos aportaría una salida a dicha situación. La Presidenta Martínez de Perón y los ministros Ivanissevich y Arrighi, por su parte, concebían que la raíz de la crisis era intrínseca al sistema. La responsabilidad sobre la pérdida de la eficacia de la transmisión y de la conversión de los instintos primarios recaía en los docentes, a quienes Ivanissevich, en reiteradas ocasiones, calificó de subversivos, huelguistas, intolerantes y amorales. Consideraba que, antes de transformar las propuestas de enseñanza, era prioritario conducir al cambio de hábitos y de ideología del magisterio.

En los discursos de Cámpora, Taiana, Benítez e inclusive en los proyectos de reforma del sistema del CFE y del Plan Trienal, la escuela y la educación eran concebidas como positivas en el desarrollo del hombre y de la Nación, estando vinculadas y dando respuestas a la formación de

\footnotetext{
${ }^{48}$ MCE, "El Ministro, Dr. Pedro José Arrighi: La Educación contra la subversión”, Boletín de Comunicaciones No 33-34. XVIII. 30/10/1975, p. 1.

${ }^{49}$ El Proyecto 13 tenía como propósito que los docentes cumpliesen con mayor eficacia el dictado de clases, la asistencia al alumno en actividades de recuperación o vocacionales, el vínculo con padres y la comunidad, el aporte a una mejor organización y funcionamiento del establecimiento escolar y la actualización y perfeccionamiento docente (MCE, Resolución $\mathrm{N}^{\mathrm{o}}$ 658, Boletín de Comunicaciones $\mathrm{N}^{\circ}$ 13. XV. 30/4/70).

${ }^{50}$ CONET, "Palabras de S.E. el Sr. ministro de Cultura y Educación Dr. Pedro José Arrighi", Boletín del CONET No 509. 3/11/1975, p. 1447.

${ }^{51}$ ARRIGHI, Pedro. "La educación en el país. Discurso pronunciado por el Sr. Ministro de Cultura y Educación con motivo del día del maestro", Bs. As., 1975.
} 
recursos humanos nacionales necesarios para el desarrollo de la economía industrial regional y nacional. El "pueblo" tenía un lugar destacado siendo artífice y promotor de cambios educativos y curriculares que responderían a las demandas regionales, no obstante, el pueblo debía formarse para la conducción. Dicho optimismo estuvo ausente en el discurso de Estela Martínez y de sus ministros, quienes expresaron permanente desconfianza del poder de transmisión de la escuela y de las capacidades de los educadores. En ocasiones dichas instituciones fueron presentadas como disruptivas de la doctrina moral y cristiana del justicialismo. En ninguno de los discursos analizados la educación media fue vinculada por estos últimos a la economía. La educación debía transformarse para recuperar su función ideológica, no económica, y por ello aspiraban su unificación en todo el territorio argentino. Durante estas gestiones se partió de concebir el fin de la educación en términos espirituales y trascendentes.

Sobre los indicadores "críticos" los ministros delinearon agendas políticas educacionales centradas en los fines que, junto a los mandatarios y a ciertos funcionarios, atribuyeron a la educación. El énfasis de Taiana en que la educación debía alistar a todos los sectores sociales para que se incorporen al trabajo efectivo y a los poderes de decisión sentó las bases de una agenda construida en forma progresiva, a partir del consenso alcanzado en numerosas reuniones del CFE. Esta agenda continúo vigente, siendo reelaborada tras la sucesión presidencial de Cámpora por Juan Domingo Perón. La enseñanza obligatoria debía ampliarse incorporando al ciclo básico de la educación media y ésta debía diversificarse otorgando a los alumnos una salida laboral. La educación técnica tuvo relevancia en las discusiones efectuadas en el marco del CFE y la capacitación técnica se planteó, en el Plan Trienal, como transversal a la enseñanza primaria y media. La universalización y modernización del sistema se reflejarían, en el ámbito de la educación técnica y media nacional, en la regionalización de la enseñanza, la innovación institucional, la transformación de la estructura curricular y en el aumento de la cantidad de establecimientos.

El fin que Ivanissevich asignó a la educación marcó también el rumbo de sus acciones. El eje de los discursos del ministro era la crisis del sistema causada por el proceder inmoral de los docentes, alumnos y por la acción de la "antiescuela." Sus esfuerzos se centraron en imponer orden y disciplina en las conductas de la comunidad educativa. La preocupación por derrotar la "antiescuela" desplazó la realización de un análisis acerca de la validez y vigencia curricular del sistema educativo, así como de los proyectos de transformación del nivel medio consensuados en el CFE e impulsados desde el CONET. Si bien manifestó su intención de seguir los lineamientos del Plan Trienal, se oponía a la regionalización de la enseñanza que el documento pautaba. La constitución de la agenda política educativa fue una construcción conjunta entre el ministro y sus funcionarios más cercanos, sin la injerencia los ministros de educación de las jurisdicciones ni de Benítez, que continuaba siendo el presidente del CONET. El CFE no sesionó durante su gestión, aunque hubo una reunión de Ministros en la que, al tiempo que las autoridades provinciales manifestaron respaldo por los derechos docentes, Ivanissevich reafirmó su postura acerca de los perjuicios causados por sus acciones.

La agenda educacional de Arrighi incorporó elementos presentes en las de sus predecesores. Apareció tanto la intención de sancionar una Ley, de respetar los lineamientos del Plan Trienal, como de luchar contra la subversión en todos los niveles del sistema educativo. Existieron algunos proyectos concretos respecto al nivel medio, aunque no fue retomado el proyecto universalista y modernizador que había comenzado a implementarse en el ámbito del CONET. Respecto a la educación técnica, se limitó a asegurar su apoyo para la conquista de un "futuro promisorio."

Con respecto a los alcances de los discursos y los proyectos que aspiraban a transformar el nivel medio en general y la enseñanza técnica en particular, es importante marcar que en el ámbito del CONET tempranamente se crearon instancias que habilitaron la participación de la comunidad educativa y de la sociedad civil en debates sobre las características que debía asumir 
la enseñanza. En este marco se derogó la ley que quitaba de la órbita del organismo un conjunto de escuelas técnicas nacionales; y se diseñó el proyecto modernizador de la enseñanza técnica que alcanzaba cambios en la estructura curricular e innovaciones institucionales. Tras el cambio de gestión ministerial dichos proyectos quedaron inconclusos y paralizados. Se llevaron adelante acciones alternativas y paliativas a las diseñadas a principios de 1974. Los cambios concretados al interior del CONET mantuvieron su estructura curricular tradicional, fortalecieron los cursos de Auxiliares técnicos (cuarto año de término) y las instancias de formación profesional vinculadas a la educación permanente. Además, se creó un régimen de excepción que habilitaba a los alumnos del ciclo superior a asistir a industrias a realizar las actividades prácticas que, hasta entonces, se realizaban en los talleres de las escuelas y un servicio vocacional que se presentaba como alternativo, frente a la imposibilidad de implementar las transformaciones estructurales del nivel medio.

En suma, se advierte que existió un proyecto que pretendía transformar radicalmente la enseñanza media del CONET que, si bien recogía las posturas de Cámpora, fue diseñado durante la gestión presidencial de Juan Domingo Perón y abandonado durante la de su sucesora. Las ideas fuerza de dicho proyecto perdieron eficacia en su concreción, lo cual da cuenta de que los intereses respecto al impulso de la educación técnica nacional no fueron uniformes, generándose cambios menores que permitieron al organismo subsistir, pero no democratizar ni modernizar la enseñanza.

\section{Bibliografía}

ABBATTISTA, María Lucía (2013). "Las políticas de la Tendencia Revolucionaria del Peronismo en el Ministerio de Cultura y Educación de la Nación (1973-1974) y los modelos latinoamericanos contemporáneos", IV Jornadas de Historia Política. Montevideo, pp. 1-21.

ABBATTISTA, María Lucía (2015). “QQué todos los chicos 'se metan', opinen, intervengan'. Un estudio sobre 'El Diario de los Chicos' publicado por el Ministerio de Cultura y Educación de la Argentina entre 1973 y 1974”. En FLIER, Patricia (Coord.) Actas de las VII Jornadas de Trabajo sobre Historia Reciente, La Plata: Universidad Nacional de La Plata, pp. 687-707.

ABBATTISTA, María Lucía (2019). Justicialismo y cultura en la Guerra Fría: El retorno de Oscar Ivanissevich al Ministerio de cultura y educación (Argentina 1974-1975). Tesis de posgrado. Universidad Nacional de La Plata. Recuperado de http://www.memoria.fahce.unlp.edu.ar/tesis/te.1801/te.1801.pdf.

BEN PLOTKIN, Mariano y ZIMMERMANN, Eduardo (Comp.), (2012). Los saberes del Estado. Bs. As.: Edhasa.

BESOKY, Juan Luis (2017). "La gestión del Ministro Ivanissevich y la derecha peronista: los 100 días de Ottalagano", Folia Histórica del Nordeste, no. 29, pp. 145-174. DOI: http://dx.doi.org/10.30972/fhn.0292435.

BOETTO, María Belén (2015). "Memoria y espacio biográfico en el peronismo. Un estudio de caso: Cómo cumplí el mandato de Perón de Héctor J. Cámpora". En FLIER, Patricia (Coord.) Actas de las VII Jornadas de Trabajo sobre Historia Reciente, La Plata: Universidad Nacional de La Plata, pp. 53-68. 
BOHOSLVASKY, Ernesto y SOPRANO, Germán (2010). El Estado con rostro humano. Funcionarios e instituciones estatales en Argentina (desde 1880 a la actualidad). Bs. As., Universidad Nacional de General Sarmiento: Prometeo Libros.

CARNAGUI, Juan Luis y ABBATTISTA, María Lucía (2004). 'La 'depuración oficial' en las políticas educativas: la gestión Ivanissevich en el Ministerio de Educación de la Nación y su impacto en la UNLP”, VIII Jornadas de Sociología de la UNLP. La Plata. Recuperado de: http://sedici.unlp.edu.ar/handle/10915/54158.

DOVAL, Delfina (2001). "Una escuela de pensamiento. Universidad y dictadura: un estilo de vida misional", en KAUFMANN, Carolina (Dir.) Dictadura y Educación. Universidad y Grupos Académicos Argentinos (1976-1983), Tomo 1, Madrid: Miño y Dávila, pp. 91-120.

DUSSEL, Inés y PINEAU, Pablo (2002). "De cuando la clase obrera entró en el paraíso. La educación técnica estatal en el primer peronismo". En PUIGGRÓS, Adriana (Dir.) Discursos pedagógicos en el imaginario social en el peronismo. 1945-1955. Bs. As.: Galerna, 107-173.

FRANCO, Marina (2011). "La 'depuración' interna del peronismo como parte del proceso de construcción del terror de Estado en la Argentina de la década del 70", A contracorriente, vol. 8, no. 3, pp. 23.54 .

FRANCO, Marina (2012). Un enemigo para la nación. Orden interno, violencia y 'subversión', 1973-1976. Bs. As.: Fondo de Cultura Económica.

FRIEDEMANN, Sergio (2016). "Transición a la dictadura durante el gobierno de Isabel Perón. El ocaso de la Universidad Nacional y Popular de Buenos Aires", Revista de la Carrera de Sociología, vol. 6, no. 6, pp. 1-34. Recuperado de: http://hdl.handle.net/11336/115792.

FRIEDEMANN, Sergio (2017). “De las Cátedras Nacionales (1967-1971) a la Universidad Nacional y Popular de Buenos Aires (1973-1974). Experiencias configuradoras de institucionalidad universitaria", Sociohistórica, no.39, e026. DOI: https://doi.org/10.24215/18521606e026.

IZAGUIRRE, Inés (2011). "La universidad y el Estado terrorista. La Misión Ivanissevich", Revista Conflicto Social, vol.4, no.5, pp.287-303. Recuperado de: https://publicaciones.sociales.uba.ar/index.php/CS/article/view/380/345.

KOC MUÑOZ, Álvaro (2014). Más que hombres sabios necesitamos hombres buenos. La expansión de la educación técnica durante el Primer Peronismo (1944-1955). Trabajo final de grado. Universidad Nacional de La Plata. En Memoria Académica. Recuperado de http://www.memoria.fahce.unlp.edu.ar/tesis/te.980/te.980.pdf.

LENCI, María Laura (1998). "La radicalización de los católicos en la Argentina. Peronista, cristianismo y revolución (1966-1971), Revista Sociohistórica. Cuadernos del CISH, no. 4, pp. 174-200.

LENCI, María Laura (1999). "Cámpora al gobierno, Perón al poder. La tendencia revolucionaria del peronismo ante las elecciones del 11 de marzo de 1973." En PUCCIARELLI, Alfredo (Ed.) La primacía de la política: Lanusse, Perón y la nueva izquierda en tiempos del GAN. Bs. As.: Eudeba. 
LENCI, María Laura (2014). "Violencia, política y terrorismo de Estado (1955-1983)." En BARRENECHE, Osvaldo. Historia de la provincia de Buenos Aires, Tomo 5: del primer peronismo a la crisis de 2001. Bs. As.: Edhasa.

MEDELA, Paula (2015). "La participación de los jóvenes militantes de la década del sesenta y setenta en la Campaña de Reactivación Educativa del adulto para la Reconstrucción (1973-1974)", XV Jornadas Interescuelas. Departamentos de Historia. Universidad Nacional de la Patagonia San Juan Bosco, pp.1-16. Recuperado de: https://cdsa.aacademica.org/000-061/881.pdf.

MOLLIS, Marcela (1991). "Historia de la Universidad Tecnológica Nacional. Una Universidad para los hombres y mujeres que trabajan," Realidad Económica, no. 99.

NAVARRO, Marcelo (2015). "Peronismo y Educación Rural en la Provincia de SaltaArgentina (1946-1955)", Revista Eletrônica de Educação, vol.9, no.3, pp.64-76. DOI: http://dx.doi.org/10.14244/198271991340.

OSZLAK, Oscar (2006). "Burocracia estatal: política y políticas públicas”, POSTData Revista de Reflexión y Análisis político, no. 11.

PALACIOS, Manuel y RODRÍGUEZ, Javier (2006). 'Para qué copiar, es preciso CREAR' (Campaña de Reactivación Educativa del Adulto para la Reconstrucción Nacional. Tesis de Licenciatura. Universidad Nacional de Córdoba.

PINEAU, Pablo (1991). Sindicatos, estado y educación técnica (1936-1968). CEAL.

PINEAU, Pablo (1997a). "La vergüenza de haber sido y el dolor de ya no ser. Los avatares de la educación técnica entre 1955 y 1983”. En PUIGGRÓS, Adriana (ed.) Dictaduras y utopías en la historia reciente de la educación argentina (1955-1983). Bs. As.: Galerna, pp. 379-402.

PINEAU, Pablo (1997b). "De zoológicos y carnavales: las interpretaciones sobre la Universidad Obrera." En CUCUZZA, Héctor (Dir.) Estudios de Historia de la Educación durante el Primer Peronismo (1943-1955). Universidad Nacional de Luján: Libros del Riel, pp.205-229.

PRONKO, Marcela (2009). "Empresarios, industriales y educación técnica en Argentina, 19201946." En ASCOLANI, Adrián (comp.) El sistema educativo en Argentina. Civilidad, derechos y autonomía, dilemas de su desarrollo histórico. Rosario: Laborde editor.

REIN, Raanan (1998). Peronismo, populismo y política. Argentina 1943-1955. Bs. As.: Editorial de Belgrano.

RODRÍGUEZ, Laura (2013). "Los católicos y la educación en el tercer peronismo (19731976)", Anuario de la Sociedad Argentina de Historia de la Educación, vol. 2, no. 14, pp. 116. Recuperado de: http://hdl.handle.net/11336/28723.

RODRÍGUEZ, Laura (2014). "La universidad durante el tercer gobierno peronista (19731976)", Revista Conflicto Social, año 7, no. 12, pp. 1-18. Recuperado de: http://sedici.unlp.edu.ar/handle/10915/50893.

RODRÍGUEZ, Laura (2015). Universidad, peronismo y dictadura. Bs. As.: Prometeo. 
ROUGIER, Marcelo y FISZBEIN, Martín (2006). La Frustración de un proyecto económico. El gobierno peronista de 1973-1976. Bs. As.: Manantial.

SERVETTO, Alicia y PAIARO, Melisa (2013). "Violencia y represión: Los discursos de María Estela Martínez de Perón (1974-1976)", Anos 90, vol.20, no.38, pp.253-283. Recuperado de: http://www.seer.ufrgs.br/index.php/anos90/article/view/29246. DOI: https://doi.org/10.22456/1983-201X.29246.

SOSA, Mariana (2016). "Desarrollo industrial y educación técnica: una estrecha relación. El caso Argentino", Revista Latino-Americana de Historia, vol. 5, no. 15, pp. 174-195. Recuperado de: https://dialnet.unirioja.es/servlet/articulo?codigo=6238599.

SPREGELBURD, Roberta (1997). "La enseñanza técnica en el nivel primario. Las misiones monotécnicas. Análisis de un caso en Luján." En CUCUZZA, Héctor (Dir.) Estudios de Historia de la Educación durante el Primer Peronismo (1943-1955). Universidad Nacional de Luján: Libros del Riel, pp. 359-399.

TOSOLINI, Mariana (2012). "Una propuesta de educación de adultos para el desarrollo nacional. La implementación de la CREAR en Córdoba", Cuadernos de Educación, vol.X, no.10, pp.1-12. Recuperado de: https://revistas.unc.edu.ar/index.php/Cuadernos/article/view/4528.

WIÑAR, David (1981). Educación Técnica y evolución social en Argentina. Bs. As.: Comisión Económica para América Latina. 